\section{Extrafeines Aerosol}

$\mathrm{s}$ eit Herbst 2006 stehen Beclometasondipropionat (BDP) und Formoterol (F) als Mikrosol in Fixkombination zur regelmäßigen Behandlung von Patienten mit Asthma zur Verfügung (Inuvair ${ }^{\circledR}$ Mikrosol). Das Mikrosol stellt eine neue Generation eines treibgasbetriebenen Inhalationssystems dar. Dabei liegen die Wirkstoffe vollständig in Lösung vor. Möglich wird dies durch die Verwendung des für die Ozonschicht unschädlichen Treibgases Norfluran (HFA-134a) und den Zusatz eines Hilfslösungsmittels. So entsteht eine homogene Lösung, die wesentlich feiner vernebelt werden kann als eine Suspension. Dies wird unterstützt durch einen weiter entwickelten Sprühkopf und optimierte physikalische Parameter des Gerätes. Das Ergebnis ist eine Sprühwolke aus besonders fein vernebelten
Partikeln, deren MMAD (medianer massenaerodynamischer Durchmesser) deutlich unter dem von Suspensionsaerosolen liegt. Auf eine Inhalationshilfe (Spacer) kann bei der Inhalation mit dem Mikrosol verzichtet werden. Darüber hinaus ist das Mikrosol so konstruiert, dass eine sanfte Sprühwolke über einen längeren Zeitraum entsteht, die

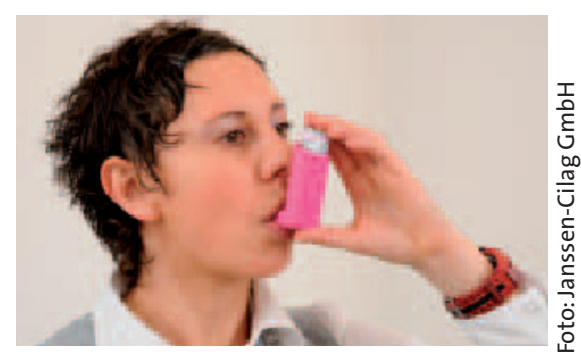

Die korrekte Inhalation ist für eine gute Asthmakontrolle extrem wichtig. sich langsam bewegt. Dies erleichtert den Patienten die Koordination während der Einatmung. Das Ergebnis bei der Inhalation des Mikrosols ist eine verminderte oropharyngeale Deposition: mehr extrafeine Wirkstoffteilchen dringen bis in die großen und die kleinen Atemwege vor. Ihre effektive Wirksamkeit und gute Verträglichkeit hat die Fixkombination in mehreren klinischen Studien unter Beweis gestellt. So ergab eine randomisierte Doppelblindstudie mit drei Parallelgruppen, dass Patienten, die mit dem Mikrosol inhaliert hatten, signifikant mehr symptomfreie Tage und Nächte und mehr Tage mit guter Asthmakontrolle hatten, als Patienten, die die beiden Wirkstoffe separat inhalierten oder eine Monotherapie mit dem herkömmlichen BDP-Druckgasaerosol erhielten.

Nach Informationen von Janssen-Cilag, Neuss

\section{Asthmabehandlung ohne Wachstumsverzögerung}

Bei Kindern mit leichtem, persistierenden Asthma führt die Therapie mit dem Leukotrienantagonisten (LTRA) Montelukast zu keiner Beeinträchtigung der Wachstumsrate, so eine aktuelle Studie [Pedersen $\mathrm{S}$ et al. Pediatric Pulmonology 2007; 42: 838-43]. Dabei war mittels Knemometrie, einem Messverfahren, das bereits kleinste Längenzunahmen über wenige Tage bis Wochen erfasst, das Längenwachstum des Unterschenkels unter der Behandlung mit Montelukast, Plazebo und dem inhalativen Steroid Budesonid untersucht worden. Bei Montelukastbehandlng wiesen die 6- bis 12-jährigen Kinder während der Behandlung eine mit der Plazebogruppe vergleichbare Wachstumsrate auf. Dagegen wurde bei BudesonidEinnahme eine signifikant verlangsamte mittlere Wachstumsrate gegenüber Plazebo gemessen $(p=0,002)$. Nach Ansicht der Autoren bestätigt die Studie die Ergebnisse früherer Studien, in denen unter der Therapie mit inhalativen Glukokortikoiden das Längenwachstum negativ beeinflusst wurde, nicht aber unter der Behandlung mit Montelukast.

Nach Informationen von MSD, Haar

\section{Kurzzeit-Immuntherapie dank Adjuvans}

$F_{\mathrm{d}}^{\mathrm{u}}$ ür viele Patienten und Ärzte hat sich die spezifische Immuntherapie (SIT) besonders in Form einer präsaisonalen Kurzzeittherapie bewährt. Die Reduktion der Injektionszahl verbessert die Compliance und damit den Therapieerfolg. Möglich wird die Kurzzeittherapie durch Adjuvantien wie Monophosphoryl-Lipid A $\left(\mathrm{MPL}^{\circledR}\right)$. Das Adjuvans verstärkt das Wirkprinzip der SIT: Während die Pollenallergene das spezifische Immunsystem stimulieren, greift das Adjuvans am angeborenen Immunsystem an und wirkt wie ein Booster auf die Allergieimpfung. Das detoxifizierte Lipid A aus der Zellwand von Salmonella minnesota bindet an Toll-like-Rezeptoren (TLR4), die wie beim Kontakt eines echten Pathogens die körpereigene Abwehr durch Stimulation von Zellfunktionen unterstützen. So fördert das Adjuvans die erwünschte Immunmodulation im Sinne einer TH1-gerichteten Immunantwort.

Die adjuvante Wirkung und gute Verträglichkeit von $\mathrm{MPL}^{\circledR}$ wurde in zahlreichen Studien sowohl im Rahmen der SIT als auch bei Impfstoffen gegen
Hepatitis oder HPV bereits ausführlich dokumentiert.

Auch im Vergleich $\mathrm{zu}$ anderen Kurzzeit-SIT zeigt MPL $^{\circledR}$ deutliche Vorteile. Der komplette Therapiezyklus pro Jahr ist mit der vierten Injektion nach drei Wochen abgeschlossen. Bereits nach dem Therapiezyklus des ersten Jahres nehmen 68,5\% der Patienten weniger Medikamente ein und die klinische Symptomatik bessert sich bei $88 \%$ der Betroffenen.

POLLINEX $^{\circledR}$ Quattro vereint die Vorteile eines Tyrosinadsorbats und des gegenüber herkömmlichen Extrakten aufgereinigten Allergoids mit dem der Boosterung der Allergieimpfung durch das Adjuvans MPL ${ }^{\circledR}$. Durch diese Kombination ist eine gut verträgliche Kurzzeit-Immuntherapie mit 4 Injektionen innerhalb von 3 Wochen pro Jahr möglich. Die Wirksamkeit und Verträglichkeit dieser Immuntherapie wurde in plazebokontrollierten Doppelblindstudien dokumentiert.

Nach Informationen von Bencard Allergie, München 